\title{
Formation of Substitution or Complementary Convergence in ICT
}

\author{
Yawei Qi ${ }^{\mathrm{a}, \mathrm{b}}$ \\ ${ }^{a}$ Jiangxi Advanced Research Center of E-commerce Engineering. $b$. School of \\ Information Technology, Jiangxi University of Finance \& Economics, Nanchang, \\ Jiangxi, China \\ qyw1227@sina.com
}

\begin{abstract}
This paper offers a framework for understanding the dynamics formation of complementary industry convergence and substitution industry convergence from the respective of industry evolution, according to convergence may involve changes in competitive and/or complementary relations between two or more industries. Based on the characteristics of ICT-intensive industry, this paper describes the relationship between industry convergence, industry symbiosis and industry evolution. Through the evolution modeling of industry system and analyzing of symbiosis stability, we find that complementary industry convergence and substitution industry convergence occur when subsystems cooperate mutually or the degree of substitution isn't strong, and complementary integration mechanisms dominate substitution mechanisms. In the complementary industry convergence, converging industry and original industry are symbiotic, and the industry system has more room for development.
\end{abstract}

Keywords: Complementary Convergence, Substitution Convergence, ICT Industry

\section{Introduction}

Convergence has been a widespread and recurrent phenomenon in industry evolution, especially in information and communication technologies (ICT) intensive sectors that coevolve with new enabling technologies the last decades, because of characteristics of ICTintensive sectors, such as infusibility, permeability, modularity and so on. Convergence was used to denote almost all aspects of the impact of the industry revolution. Now, this trend has gradually extended to other traditional industries. The popular image used to illustrate this convergence was three or four circles gradually moving into each other $(e . g$., Negroponte cited in Brand 1987 and Wirtz 2001). They usually represented IT, telecom, media and consumer electronics industries. The four industries were expected to merge into one big blob "the converging industries", in which the old industry barriers were teared down and where everybody would compete with everybody. The aim of this article is to trace the roots of the concept, and from the perspective of industry system evolution attempt to analyze dynamic formation and type of convergence.

A working definition of convergence could be something like: "merging of hitherto separated markets, removing entry barriers across industry boundaries" [15]. The convergence phenomenon implies the deconstruction of existing value chains, transforming industry boundaries and creating new competitive spaces. The convergence article by Greenstein \& Khanna takes their point of departure in models of technological change and industry evolution, and they suggest a model with two types of convergenceconvergence in substitution and convergence in complement [6]. When two different product classes start to share features that will make them interchangeable for customers, we will have a convergence of substitutes. If two product classes work better together than by themselves we have convergence of complements. References $[9,11,12]$ further introduced the categories demand side and supply side convergence, and pointed out that 
supply side convergence has to do with technological functionality. A supply side convergence in substitutes occurs when different technological capabilities become similar and can satisfy then same set of needs. If there is a supply side convergence in complements, different technologies are brought together to create new kinds of opportunities. A demand side convergence in substitution arises when the needs of different consumer segments become similar. Complementary convergence on the demand side arises when different but related consumer needs are met by product bundling.

Innovation is the essence of industry convergence, thus the revolution of industry systems during convergence is a re-action to the outside force of industry restructuring. In the case of inter-industry interactive convergence, the main significant distinctive boundaries between economic units are broken by closely interdependent nature, appearing symbiotic form. The keys to industrial symbiosis are collaboration and the synergistic possibilities offered by geographic proximity [4]. References [2, 3] offer an important theoretical perspective suggesting how the emergence of industry symbioses based on the exploitation of win-win situations among area firms could lead to a form of organization that embraces sustainable industrial development. References [14] argued that several cases of industrial symbiosis, and in the cases the industry convergence is considered fruitful from the companies' point of view. References $[7,16]$ introduced the symbiosis theory to the industrial economic, and described industry symbiosis activity pattern and system type and basic problems through the analysis of the connotation and basic character. And Logistic equation was applied to describe dynamically the evolution process of industry cluster. Evolution stages of traditional industries and strategic emerging industries convergence development consist of three stages, including corresponding stage of each other, coordinate development stage and alternative stage [10]. References [8] analyzed the synergy evolution law of emerging technologies and emerging industries, and dynamic evolution of knowledge learning and diffusion, demand space and structure change.

The previous literatures lack of comprehensive description of relationship among industry symbiosis, convergence and evolution, and quantitative analysis of formation of industry convergence. We claim that evolution is strongly driven by competition and cooperation, based on interaction and mutual dependence among organisms. Symbiotic and convergence involve changes in competitive and/or complementary relations between two or more industries, urging evolution of industry systems together. We use Logistic model to analyze the formation of industry convergence in the process of industry evolution.

Industry convergence is in nature economic histology phenomenon, being selforganizing. Although the division functions of each symbiotic unit were varied, but they can achieve effective convergence, interaction and coordination under the drive of common interest, and develop into another organization beyond market and business form. Symbiosis relation of industry convergence focus on industrial innovation and business connections in the process of value added. Convergence in the sense of symbiotic is to create value for the basic premise. From the point of achieving ways, complementarities in technologies, supply and demand of products, and combination of business modules can promote the formation of convergence from the perspective of evolution. The interaction between industries is the material basis of continual promoting the symbiotic relationship of industry convergence behavior. At the same time pay attention to state stability in the process of industry evolution, which is able to determine whether industry convergence arises.

This paper is organized as follows. In the next section, the symbiotic evolution of ICTintensive industry system is described with logistic model. In Section III, determine the stability of the symbiotic points, and the formation of complementary convergence and substitution convergence. Finally, we conclude the paper. 


\section{Formatting your Paper}

\subsection{Motivate for IT Industry Systems Evolution}

Industry system is open, and subsystems involve physical exchange of materials, energy through a variety of correlating ways, forming certain structure and organization, achieving a certain function. In this paper, the way linking subsystems was divided into two categories: competition and cooperation. Assumed that subsystems can achieve symbiosis through similar resources sharing or heterogeneous resources complement, forming industry ecosystem.

Industry system is mainly affected by three factors in the course of its evolution: individual fitness, market environment, as well as correlation with other industries. Openness, non-linear and non-equilibrium of industry system are conditions of selforganizing evolution. Technological innovation driven by profit changes the income elasticity of products and causes differences in productivity. Thus result in alternation of leading industry, and lead to restructuring, reorganizing and upgrading of industrial structure, which is the essence of self-organizing evolution. The characteristics of information industry make the evolution of industry system is particularly special. Convergence and symbiosis will be the trend of the evolution. Industrial symbiosis takes business modules as accrete unit. The approach mode and mechanisms between the modules is referred to as symbiotic interface, which is medium, channel or carrier of material, information and energy transmission between symbiosis unit, and is the basis of symbiotic relationship formation and development, The operational mechanism between modules specifically refers to internal interface, and technical standards, design principles etc. belong to the external interface, of which openness increases with the harmonization degree of technical standards increasing.

The extensive application of general technology, such as digital technology, makes different companies in information systems more closely related, and promotes convergence of technical standards and design principles, resulting in the evolution of system trend to convergence. Network effect makes it easier for innovative technology to diffuse and absorb, so that enterprises have a common technology basis, and the phenomenon of technology convergence appears. Competitive fiercely market environment promotes collaborative mode of industrial ecosystem based on module happen and develop. Module is an open structure, and in the case of the same standards and design principles, a new module through operations of modules decomposing and restructuring promotes emergence of new enterprises and sub-industries, thus leading to the rationalization and optimization of the information industrial structure. The interaction between different modules increases collaboration degree among different enterprises in the ecosystem. At the same time, modular operating can innovate closely around customer needs. Today, consumer demand tends to diversification and integration, spurring the company to carry out institutional collaboration and integration, and produce innovative products based on converging markets, which is a sign of industry convergence. On the one hand, convergence leads to additional value-added opportunities within these markets, allowing new intermediaries to enter the market. On the other hand, traditional entry barriers of these markets disappear and new forms of competition and substitution for existing suppliers and products emerge. We take mobile services as an example for a promising innovative field of added value creation in the converging markets. In order to increase competitiveness, two utilization scenarios within the mobile service market are emphasized: Portable internet access via mobile terminals and mobile services developed to satisfy the particular needs of mobile customers. In conclusion, the collaborative mode of industry ecosystem has a strong innovative capacity. Enterprises, actively participating 
in technological competition and cooperation, are nonetheless exchanging and sharing knowledge with other enterprises, making the barriers of industries fuzzy, complementary convergence and substitution convergence arise.

\subsection{Evolution Model of Industry Ecosystem}

Logistic model is commonly used to describe the laws of economic or population growth, and sometimes use it to depict the interaction between enterprises and groups, so describing symbiotic evolution of industry system with Logistic model is quite appropriate. For the sake of brevity, it is assumed that in an open environment, the industry ecosystem is composed of two subsystems $F_{1} 、 F_{2}$, which represent merging industry and existing industry respectively. The evolution model of industry ecosystems is:

$$
\left\{\begin{array}{l}
\frac{d X_{1}}{d t}=f_{1}\left(X_{1}, X_{2}\right)=\alpha_{1} X_{1}\left(N_{1}-X_{1}-\beta_{12} X_{2}\right)-\delta_{1} X_{1} \\
\frac{d X_{2}}{d t}=f_{2}\left(X_{1}, X_{2}\right)=\alpha_{2} X_{2}\left(N_{2}-X_{2}-\beta_{21} X_{1}\right)-\delta_{2} X_{2}
\end{array}\right.
$$

Where $X_{1} 、 X_{2}$ respectively denotes the products number of subsystems; $\alpha_{1} 、 \alpha_{2}$ denotes the growth rate respectively, having something with innovation; $N_{1} 、 N_{2}$ represents the largest potential market capacity; $\delta_{1} 、 \delta_{2}$ represents the average consumption rate; $\beta_{\mathrm{ij}}(i, j=1,2, i \neq j)$ represents the impact of subsystems $F_{j}$ on the subsystem $F_{\mathrm{i}}$, and its value can be negative or positive. $\beta_{\mathrm{ij}}=0$ shows there is no correlation between subsystems; $\beta_{\mathrm{ij}}<0$ denotes the relationship between subsystems is alternatively competitive; $\beta_{\mathrm{ij}}>0$ denotes subsystems are in complement.

$$
\left\{\begin{array}{l}
\frac{d X_{1}}{d t}=f_{1}\left(X_{1}, X_{2}\right)=\alpha_{1} X_{1}\left(P_{1}-X_{1}-\beta_{12} X_{2}\right) \\
\frac{d X_{2}}{d t}=f_{2}\left(X_{1}, X_{2}\right)=\alpha_{2} X_{2}\left(P_{2}-X_{2}-\beta_{21} X_{1}\right)
\end{array}\right.
$$

Where $P_{1}=N_{1}-\frac{\delta_{1}}{\alpha_{1}}, P_{2}=N_{2}-\frac{\delta_{2}}{\alpha_{2}}$, equation (2) indicates that the impact of $F_{j}$ on evolution of $F_{i}$ is achieved through competing for customers of subsystem $F_{i}$, reflecting the coefficient $\beta_{\mathrm{ij}}$. We order:

$$
\begin{aligned}
& \frac{d X_{1}}{d t}=f_{1}\left(X_{1}, X_{2}\right)=0 \\
& \frac{d X_{2}}{d t}=f_{2}\left(X_{1}, X_{2}\right)=0
\end{aligned}
$$

So get four evolution steady points of information industry:

$$
\begin{gathered}
B_{1}(0,0), B_{2}\left(0, P_{2}\right), B_{3}\left(P_{1}, 0\right), B_{4}\left(\frac{P_{1}-\beta_{12} P_{2}}{1-\beta_{12} \beta_{21}}, \frac{P_{2}-\beta_{21} P_{1}}{1-\beta_{12} \beta_{21}}\right)\left(\beta_{12} \beta_{21} \neq 1\right) \\
B_{4}\left(X_{1}^{0}, X_{2}^{0}\right)\left(\beta_{12} \beta_{21}=1, X_{1}^{0}+X_{2}^{0}=N_{1}-\frac{\delta_{1}}{\alpha_{1}}=N_{2}-\frac{\delta_{2}}{\alpha_{2}}\right) .
\end{gathered}
$$

Where, the steady point $B_{1}$ means two industries perish together through fierce competition; the steady points $B_{2}$ and $B_{3}$ mean that the two industries merge into the converging industry with the help of competition or collaboration; the steady point $B_{4}$ means that industries have a reciprocal impact, progress in one sector promoting progress in the other. 
For any steady point, characteristic matrix $B$ of evolution is:

$$
B=\left[\begin{array}{ll}
\frac{\partial f_{1}}{\partial X_{1}} & \frac{\partial f_{1}}{\partial X_{2}} \\
\frac{\partial f_{2}}{\partial X_{1}} & \frac{\partial f_{2}}{\partial X_{2}}
\end{array}\right]=\left[\begin{array}{cc}
\alpha_{1}\left(P_{1}-2 X_{1}-\beta_{12} X_{2}\right) & -\alpha_{1} \beta_{12} X_{1} \\
-\alpha_{2} \beta_{21} X_{2} & \alpha_{2}\left(P_{2}-2 X_{2}-\beta_{21} X_{1}\right)
\end{array}\right]
$$

The steady point $B_{1}$ suggests information industry does not withstand the test, and will not lead to the realization of convergence, therefore, we only consider three other steady points.

Putting $B_{2}\left(0, P_{2}\right)$ into equation (3), we get two eigenvalues:

$$
\lambda_{1}=\alpha_{1} P_{1}-\alpha_{1} \beta_{12} P_{2}, \lambda_{2}=-\alpha_{2} P_{2} .
$$

Similarly, the eigenvalues of $B_{3}\left(P_{1}, 0\right)$ are: $\lambda_{1}=-\alpha_{1} P_{1}, \lambda_{2}=\alpha_{2} P_{2}-\alpha_{2} \beta_{21} P_{1}$.

When $\beta_{12} \beta_{21} \neq 1$, the corresponding eigenvalues of $B_{4}$ are:

$$
\lambda_{1}=\frac{-b+\sqrt{b^{2}-4 c}}{2}, \lambda_{2}=\frac{-b-\sqrt{b^{2}-4 c}}{2}
$$

where $b=\frac{\alpha_{1}\left(P_{1}+\beta_{12} P_{2}\right)+\alpha_{2}\left(P_{2}+\beta_{21} P_{1}\right)}{1-\beta_{12} \beta_{21}}$

$$
c=\frac{\alpha_{1} \alpha_{2}\left[\left(P_{1}+\beta_{12} P_{2}\right)\left(P_{2}+\beta_{21} P_{1}\right)-\beta_{12} \beta_{21}\left(P_{1}-\beta_{12} P_{2}\right)\left(P_{2}-\beta_{21} P_{1}\right)\right]}{1-\beta_{12} \beta_{21}}
$$

When $\beta_{12} \beta_{21}=1, X_{1}^{0}+X_{2}^{0}=N_{1}-\frac{\delta_{1}}{\alpha_{1}}=N_{2}-\frac{\delta_{2}}{\alpha_{2}}$ denotes a straight line, and the corresponding eigenvalues are: $\lambda_{1}=0, \lambda_{2}=-\left(\alpha_{1} X_{1}^{0}+\alpha_{2} X_{2}^{0}\right)$.

\section{Formaton of Convergence}

This chapter will discuss the stability of symbiotic, that is, determine whether convergence can eventually realize, and whether converging industry can co-exist with the existing industry. Two or more industries 'come together' through substitution or complementary relations.

\subsection{Formation of Substitution Convergence}

At this point, $0<\left|\beta_{\mathrm{ij}}\right|<1$ indicates that the industry system is symbiotic partly (substitution symbiosis partly, or complementary symbiosis partly). When $\beta_{i j}$ is positive, two industries is substitution mutually. As for subsystem $F_{i}(i=1,2),\left(P_{i}-\beta_{i j} P_{j}\right)$ is the actual market demand.

$\frac{P_{i}}{P_{j}}>\beta_{i j}$ implies a market demand for subsystem $F_{i}$ is more than impact of other subsystem $F_{j}$. In this case, for eigenvalues of $B_{2}, \lambda_{1}>0, \lambda_{2}<0$; for eigenvalues of $B_{3}, \lambda_{1}>0, \lambda_{2}>0 . B_{2}, B_{3}$ are saddle points, and are unstable, which mean not bringing substitution convergence. Due to $\left.\left(P_{1}+\beta_{12} P_{2}\right)\left(P_{2}+\beta_{21} P_{1}\right)\right\rangle\left(P_{1}-\beta_{12} P_{2}\right)\left(P_{2}-\beta_{21} P_{1}\right)$, so $c>0, b>0$, we drive eigenvalues of $B_{4}: \lambda_{1}<0, \lambda_{2}<0$. From this, we can see steady point $B_{4}$ is stable. Substitution convergence occurs, converging industry partly substitute existing industry, and the converging industry can coexist with existing industry. When the system is substitution, total output of industry system is $X^{0}=\frac{P_{1}-\beta_{12} P_{2}}{1-\beta_{12} \beta_{21}}+\frac{P_{2}-\beta_{21} P_{1}}{1-\beta_{12} \beta_{21}}$, and $X^{0}>P_{1}, X^{0}>P_{2}$. Converging industry make use of environmental resources more 
effectively on a wider scale, and produce more diversified convergence products and services on form and content, promoting the development of traditional industries.

In the competitive industrial ecosystem, if the market demands for subsystem $F_{i}$ is less than substitute of other subsystems, that is $P_{i}<\beta_{i j} P_{j}$. We can see that the corresponding steady point $B_{2} 、 B_{3}$ is stable. Industry convergence through substitution occurs when the growth of an asset or activity in one industry reduces the marginal value of a corresponding asset or activity in another industry and eventually, in its ultimate form, leads to the full replacement of the latter by the former. So existing industry appear, that is, converging industry with the existing industry can't coexist [13].

From the analysis of steady-state solution, we can see that when the degree of correlation of subsystem $F_{i}$ and $F_{j}$ locates in different range, evolution path of system is different. In particular, when $0<\beta_{i j}<\frac{P_{i}}{P_{j}}$, interaction between subsystems will not promote substitution convergence occurring, and industry system can realize symbiosis. When $1 \geq \beta_{i j}>\frac{P_{i}}{P_{j}}$, substitution convergence occurs. Therefore, $\beta_{i j}=\frac{P_{i}}{P_{j}}$ is the mutation point and bifurcation point of evolution. In a word, the evolution of industry system is meaningful in enclosed area surrounded with four steady points, and evolutes toward the state of $B_{4}$.We give schematic diagram of several possible phase orbits.

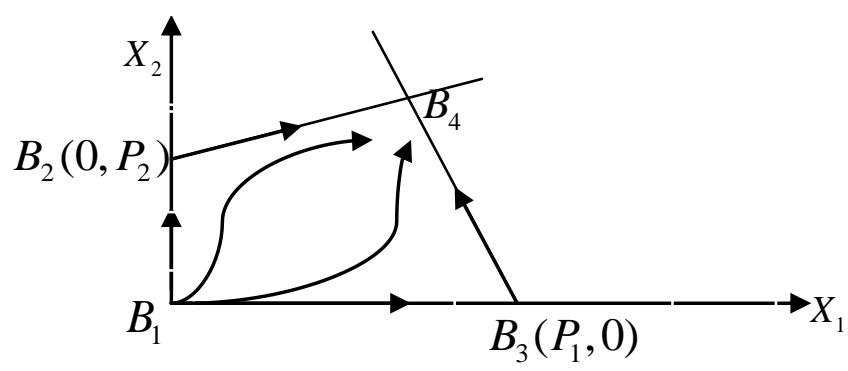

Figure 1. Schematic Diagram of Possible Phase Orbits

If $\beta_{12}=\beta_{21}=1$, we can see that $B_{2}$ is steady point depends on $P_{2}>P_{1}$. Instead, the opposite is true. While $B_{4}$ is critical stable. When there is perfect competition alternative between the subsystems, substitution convergence occurs. For example, the development of mobile technology has led to mobile phones and pagers convergence in substitution.

\subsection{Formation of Complementary Convergence}

Complementary relations between two industries occur if the growth of one asset or activity in one industry increases the returns to expanding another asset or activity in a different industry. That is, $-1<\beta_{i j}<0 .\left(P_{i}-\beta_{i j} P_{j}\right)$ is the market saturating capacity, while $\left(P_{i}+\beta_{i j} P_{j}\right)$ is the actual market demand. Define $\gamma_{i}=\frac{P_{i}+\beta_{i j} P_{j}}{P_{i}-\beta_{i j} P_{j}}$ as the sales rate of a subsystem.

Complementary convergence occurs when complementary relations imply the integration or merging of assets or activities in two industries [12]. Complementary convergence and divergence involve changes in boundaries and relations between two or more industries characterized by vertical input-output relations or other forms of complementary relations. Such changes may either lead to convergence of two or more 
hitherto distinctive industries, or the divergence of one industry into one or more component-, service or IP-based industries. Especially due to common digital foundation of ICT-intensive products and technologies, complementary convergence and divergence are pervasive forms of industry dynamics. When the sales rate of subsystems self is more than the impact of other subsystem, that is $\gamma_{i}>\left|\beta_{i j}\right|$, we drive $B_{2}, B_{3}$ are unstable, and $B_{4}$ is stable. Complementary convergence occurs, and the original industries don't disappear. The converging industry can coexist with existing industry. When the system is complementary, total market demand of new industry system is $X^{0}=\frac{P_{1}-\beta_{12} P_{2}}{1-\beta_{12} \beta_{21}}+\frac{P_{2}-\beta_{21} P_{1}}{1-\beta_{12} \beta_{21}}$, compared to the original total market demand $\left(P_{1}+P_{2}\right)$, incremental demand is $\Delta=-\beta_{21} P_{1}\left(1-\beta_{12}\right)-\beta_{12} P_{2}\left(1-\beta_{21}\right)>0$. Complementary industry convergence expands the market capacity.

Minicomputers and microcomputers were for long distinctive niches at safe competitive distance to the mainframe computer industry. However, the trajectories of these niches lead to new computer systems (PC and Client/Server systems) that were in some respects superior and in other respects complementary to mainframes and eventually implied the eclipse of classical computer industry [1], reflecting dynamics of both competitive and complementary convergence.

Two industries are completely complementary if $\beta_{12}=\beta_{21}=-1$, and an industry's survival and development is closely related to another industry. In this case, $B_{1}, B_{2}$ , $B_{3}$ are saddle points, and $B_{4}$ is critical stable. When subsystem is completely complementary to each other, complementary convergence possibly occurs and the needs of consumers are fully meet.

\section{Conclusions}

Convergence has been a trend in many industries, especially in ICT-intensive sectors. This paper firstly analyzes the relationship among convergence, symbiosis and evolution. In the process of industry convergence based on information industry development, the exchange of material, energy and information through the symbiotic interface between subsystems is the key to symbiosis. Convergence with the popularity of symbiosis motivate corporate to move and diversifications. Convergence plays an important role in the evolution of many industries, and industry convergence and industry evolution are interdependent mutually. Industry convergence implies competitive and/or complementary relations between different industries with different rules of development and results in the formation of new industries and the merging, de-merging and eventual demise of existing industries.

Industry convergence can take the shape of either substitution or complementary integration of assets (products and technologies) or activities (forms of buyer supplier relations) in two or more industries. We analyze dynamics formation condition of complementary industry convergence and substitution industry convergence from the evolution model of industry system and analysis of symbiosis stability, and find that convergence in substitution and complementary can be achieved when subsystems cooperate mutually or the degree of substitution isn't strong. Industry convergence, and in particular complementary convergence, represents the dominant pattern of industry evolution, complementary integration mechanisms dominate substitution mechanisms. In this case, complementary integration is considered fruitful from the industry system's point of view. The converging industry and original industry can be symbiotic, and resources are made use of more efficiently, increasing product demand. In the process of 
evolution, market share is the bifurcation point. It is concluded that great care should be taken in boundary conditions depending on the case and the nature of the study.

\section{Acknowledgements}

This research was supported by the Natural Science Foundation (No. 71463023) of China, Humanities and Social Sciences Foundation by the Ministry of Education of China (No. 14YJCZH114), and the School Subject (No. 06102015) of Jiangxi University of Finance and Economics.

\section{References}

[1] T. F. Bresnahan and S. Greenstein, "Technological competition and the structure of the computer industry", The Journal of Industrial Economics, vol. 1, (1999).

[2] L. W. Baas and F. A. Boons, "An industrial ecology project in practice: exploring the boundaries of decision-making levels in regional industrial systems", Journal of Cleaner Production, vol. 4, no. 12, (2004).

[3] J. N. Brings, "Industrial symbiosis in Kalundborg, Denmark. A quantitative assessment of economic and environmental aspects", Journal of Industrial Ecology, vol. 10, no. 12, (2006).

[4] M. R. Chertow, "Industrial symbiosis: literature and taxonomy", Annual Review of Energy and Environment, vol. 4, no. 25, (2000).

[5] J. F. Christensen, "Industrial evolution through complementary convergence: the case of IT security", Industrial and Corporate Change, vol. 1, no. 20, (2011).

[6] S. Greenstein and T. Khanna, "What does industry convergence mean", Boston: The President and Fellows of Harvard Press, (1997), pp. 201-226.

[7] H. Xiao-Peng, "Theory definition and internal mechanism of industry symbiosis", China Industrial Economics, vol. 9, (2008).

[8] H. Lu-Cheng and Y. Xue-Jun, "Synergy evolution of emerging technologies and emerging industries", Science \& Technology Progress and Policy, vol. 3, (2014).

[9] J. Lind, "Convergence: History of term usage and lessons for firm strategists", ITS 15th Biennial Conference, Berlin, Germany, (2004).

[10] L. Li-Jun and Y. Bin-Bin, "Integrated evolution of traditional industries and strategic emerging industries and government behavior: theory and evidence", China Soft Science, vol. 5, (2012).

[11] J. Pennings and P. Puranam, "Market convergence \& firm strategy: new directions for theory and research", ECIS Conference, The Future of Innovation Studies, (2001).

[12] N. Stieglitz, "Digital dynamics and types of industry convergence: the evolution of the handheld computers market", In The Industrial Dynamics of the New Digital Economy, Christensen JF, Maskell P (eds). Edward Elgar: Cheltenham, (2003).

[13] N.Stieglitz and K. Heine, "Innovations and the role of complementarities in a strategic theory of the firm", Strategic Management Journal, vol. 7, no. 28, (2007).

[14] A. Wolf, M. Eklund and M. Sderstrom, "Developing integration in a local industrial ecosystem-An explorative approach", Business Strategy and the Environment, vol. 6, no. 13, (2007).

[15] D. Yoffie, "Competing in the age of digital convergence", California Management Review, vol. 4, no. $38,(\mathbf{1 9 9 6 )}$.

[16] Z. Hao, "Enterprises cluster co-existence model and stability analysis", Systems Engineering, vol. 4, (2003).

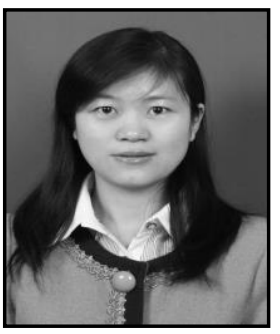

Yawei Qi, 1984.01.29, Shandong

Current position, grades: Teacher, lecturer

University studies: Applied Economics

Scientific interest: Theory and application of Econometrics

Publications: 16 papers and Two works

Experience: Teacher of Jiangxi University of Finance \&

Economics. PhD of Jiangxi University of Finance \& Economics 